\title{
Commentary
}

\section{Management of palatal fistulae}

\section{Michael H. Carstens}

Associate Professor of Plastic Surgery, Saint Louis University, Missouri, USA

Address for correspondence: Dr. Michael H. Carstens, Department of Plastic Surgery, Saint Louis University, Missouri, USA. E-mail: michael.carstens@mac.com

his retrospective analysis presented by the Sri Ramachandra craniofacial team raises several important issues for all cleft surgeons. First, palatal fistulae continue to be a common management problem. Second, the classification systems currently applied to cleft lip and palate are empiric, archaic, and misleading. Third, without a classification system based on embryologic mechanism, accurate appreciation of outcomes data generated by cleft palate (CP) surgery (i.e., surgical complications and speech) will remain chaotic. Fourth, the distribution pattern of fistulae is directly related to the technical problems engendered by the primary surgery. Fifth, the low incidence of fistulae reported in the Sri Ramchandra Medical College (SRMC) patients vs. those referred from

\begin{tabular}{|l|l|}
\multicolumn{3}{c}{ Access this article online } \\
\hline Quick Response Code: & $\begin{array}{l}\text { Website: } \\
\text { www.ijps.org }\end{array}$ \\
\cline { 2 - 2 } & Dol: \\
\hline
\end{tabular}

Indian Journal of Plastic Surgery January-April 2011 Vol 44 Issue 1 other location points out the importance of surgical protocol and concentration of expertise. Sixth, the best treatment for palate fistula is prevention using dissection techniques based on developmental anatomy of the cleft and executed with precision. Seventh, established fistulae require the same careful embryologic analysis; closure unusually involves a reapplication of techniques that should have been employed at the primary operation.

Fistula rates reported in the literature range as high as $58 \%$ with a recurrence rate of nearly 33\%.[1] These by definition result from a prior surgical procedure. Does particular operation predispose to fistula formation? The problem is complicated by a worldwide lack of consensus regarding a treatment protocol. Surgical management has been studied from the standpoint of timing (early vs. late) ${ }^{[2,3]}$ and staging ${ }^{[4,5]}$ but not by embryologic mechanism. In short, raw data regarding what factors are associated with a fistula is difficult to interpret.

The problem comes from our inability to stratify clefts by embryologic mechanism. A CP resulting from a small 
vomer is different from one involving a deficient palatine bone. Soft palate clefts involving the tensor alone can have perfectly good constrictor function. A submucous cleft with global involvement of third arch muscles will have a radically different prognosis. In short, continuing to compare apples with oranges makes outcome analysis unreliable.

CPs come in different flavours. Anatomic defects seen in CP involve distinct developmental fields, each of which contains a variety of tissues: epithelium, dermis and submucosa, cartilage, bone, fascia, and muscle. Regardless of content, the tissues in each developmental field share a common neurovascular supply. Knowledge of the nerve supply to any given field allows one to determine precisely where it originates in the embryo..$^{\mid 6,7]}$

Two types of mesenchymal structures contribute to development of the hard and soft palate: neural crest bone fields and paraxial mesoderm muscle fields. These, in turn, can be related to the developmental sector of the CNS that supplies innervation. These sectors are known as neuromeres and their individual nomenclature comes from the names applied to the original three-part embryonic brain.

All components of the hard palate, both hard and soft, are supplied by nerves having their nuclei within the hindbrain or rhombencephalon. The bones and muscles of the palate are developmentally linked to 12 rhombomeres ( $\mathrm{r} 0-\mathrm{r} 11)$. For example, all bones of the palate are innervated by V2, the nucleus of which resides in the second rhombomere. Thus, neural crest cells destined to become premaxilla, vomer, inferior turbinate, prepalatine, palatine, and maxilla all rise in the neural fold directly above r2. Clefts involving the hard palate result when deficits in one or more of these populations create palatal bone fields that are malformed, small, or downright absent.

In a similar way, all striated craniofacial muscles develop from paraxial mesoderm (PAM) lie down along both sides of the CNS. PAM begins with the hindbrain at the level of r0 (the future isthmus and pituitary) and extends backward all the way to the tail. Just as the neuraxis is segmented into neuromeres, PAM is also segmented into hollow balls called somitomeres. $\mathrm{Sm} 1-\mathrm{Sm} 7$ give rise to the extraocular muscles and the muscles of pharyngeal arches $1-3$. Since these somitomere are incompletely separated, a potential confluence of the mesenchyme exists. This explains why the muscles of the second arch migrate widely over (and under) those of the first arch.

Beginning with the eighth somitomere, the mesoderm undergoes a further rearrangement. The result is that Sm8 becomes the first somite. All somites are surrounded by epithelium and contain subunits: bone-forming sclerotomes, dermis-forming dermatomes, and muscleforming myotomes. The muscle content of all mammalian somitomeres and somites has been mapped out. Thus, $\mathrm{Sm} 4$ provides tensor veli palatini. All remaining soft palate muscles (including superior constrictor) arise from $\mathrm{Sm} 7$. Clefts of the soft palate result when deficits in one or more of these mesodermal populations create muscle fields that are malformed/mal-innervated, small, absent, or downright absent.

Fistulas are man-made creations. They result from inadequate surgical dissection of soft tissues from the margins of the cleft. Two anatomic sites are typically involved. Trouble is most often seen along the primary palate, with overlap into the adjacent secondary palate. In the SRMC series, anterior hard palate fistulae were encountered in $77.5 \%$ cases. In most cases, this results from failure to achieve control of the anterior nasal floor. Primary surgery, be it for the cleft palate or for an associated cleft lip, is the "golden opportunity" to access the anterior palate. After the primary repair (and with further growth) approximation of the palate margins makes dissection of the nasal layer more difficult.

The other common site of fistula formation is the posterior margin of the hard palate. Here, once again, the problem is one of the inadequate mobilization. The horizontal plate of the palate bone, normally having a rectangular shape, is often attenuated into a triangle. The mucoperiosteum of the nasal floor is tractioned forward. Confluence of soft tissues between the vomer and the hard palate presents difficulties as well. The stakes get higher when a Furlow Z-plasty is used for a primary palate repair. Clefts of moderate to severe width do not lend themselves well to this technique. Tissue loss and breakdown at the junction pose challenges for the most experienced operator.

SRMC reports one-year follow-up in 2067 patients with

Table 1: Neuromeric classification of palate bones and muscles

For our purposes, first arch muscles arise from Sm4, second arch muscles arise from Sm6, while those third arch belong to Sm7. Middle constrictor and cricothyroid arise from Sm8, while inferior constrictor and internal laryngeal muscles arise from early Sm9. Shortly thereafter, reorganization of Sm8-Sm11 into occipital somites S1-S4 gives us the four sources of tongue muscle. 
First rhombomere (ethmoid): Unilateral/bilateral perpendicular plate (holoprosencephaly) $>1$. Complete unilateral CP with ipsilateral central incisor absent; 2. Complete bilateral $\mathrm{CL}(\mathrm{P})$ with absent premaxilla.

First rhombomere (medial pterygoid plate): Altered dynamics of tensor veli palatini; also seen in syndromes affecting ethmoid complex.

Second rhombomere (premaxilla): Alveolar CP (primary) hard palate.

Second rhombomere (vomer): Midline ("isolated") CP.

Second rhombomere (premaxilla + vomer): Alveolar + palatal shelf CP; ipsilateral central incisor present

Second rhombomere (prepalatine ... the palatine bone secondarily affected): Palatal shelf CP (secondary hard palate)

Second rhombomere (inferior turbinate ... prepalatine secondarily affected): Tessier 3 cleft

Second rhombomere (palatine): Posterior notch of hard palate (muscles malinserted but otherwise normal mesenchyme)

Third rhombomere (mandible): Pierre Robin

Second + third + fourth + fifth rhombomeres: Craniofacial microsomia

Second + third + fourth + fifth + sixth + seventh rhombomeres: Treacher-Collins-Franceschetti ( $r 4-r 5>$ facial nerve, r2-r3 anterior pinna, r6r7 posterior pinna)

Fourth somitomere (first arch > tensor veli palatini): Relatively good palate lift, poor middle ear function, good pharyngeal contraction

Sixth somitomere (second arch > muscles of facial expression): Involvement in clefts with associated second arch abnormalities, that is, craniofacial microsomia

Seventh somitomere (third arch > levator veli palatini, uvulus, palatopharyngeus, palatoglossus, superior constrictor): Poor palate lift, middle ear function OK, poor pharyngeal contraction.

only 37 fistulae (1.9\%). This number is no accident. First and foremost, reduction of fistulae by SRMC team is designed with extensive experience and careful dissection. However, it must be stated that any cleft surgeon can achieve similar results, provided that she/he slows down and targets dissection of the anterior and posterior palate margins to achieve a tension-free, watertight closure.

With these results as a benchmark, what steps can be taken to prevent fistula formation? Recalling the sporting dictum "the best defense is a good offense," the goal should be to seal up the nasal lining of the anterior palate. This is done by dissecting out, and uniting, two adjacent neuroangiosomes: medial sphenopalatine and lateral sphenopalatine. This concept lies at the heart of DFR (developmental field reassignment) cleft surgery. ${ }^{[8,9]}$

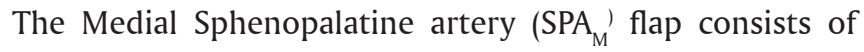
mucoperiosteum of the vomer in continuity with that of the posterolateral "shoulder" of the premaxilla. These two components are, in turn, continuous with the soft tissues of the non-philtral prolabium (NPP). Lateral Sphenopalatine artery flap $\mathrm{SPA}_{\mathrm{L}}$ is the mucoperiosteal lining of the nasal hard palate. After $\mathrm{SPA}_{\mathrm{M}}$ and $\mathrm{SPA}_{\mathrm{M}}$ are united, NPP is folded sideways 90 degrees and sutured anteriorly, thus lengthening the nasal floor. The NPP flap contains mesenchyme originally designated for the premaxilla, specifically the lateral incisor zone and frontal process zones.

Elevating the mucoperiosteum from the nasal aspect of the palate is like putting the bell on the tiger; it sounds easy in theory but proves difficult in execution. Intraoral access gets trickier the more anterior one proceeds. Extraoral access proves a useful alternative. An incision in the lateral nasal wall just anterior to the inferior turbinate (and behind the nostril sill) accomplishes two goals: (1) external rotation of the nostril sill and (2) direct access to the piriform rim and the palatal shelf. One first slides an amalgam packer or angled elevator posteriorly just beneath the turbinate anterior and proceeds posteriorly all the way to the soft palate. Next, one takes the dissection medially elevating the nasal mucoperiosteum from lateral to medial. Finally, one incises along the cleft margin to elevate the flap.

Note that the above manoeuvres can be readily accomplished during initial surgery in three scenarios: (1) complete closure of the $\mathrm{CP}$ (with cleft lip repair performed secondarily; (2) as part of a lip adhesion procedure for a wide alveolar cleft; and (3) during DFR (developmental field reassignment) cleft lip repair.

Turning our attention to the posterior margin of the hard palate, let us see how the nasal lining can be mobilized. Achieving a complete release may require subperiosteal elevation of lateral nasopharyngeal mucosa. This is accomplished by carefully freeing the nasal lining from the posterior margin of the palatine bone. One then proceeds laterally until encountering the medial pterygoid plate. Here the subperiosteal elevation follows the pterygoid cephalad toward the cranial base. If required, the release 
can be taken up to the nasal roof. The oral tissues are often tethered with the greater palatine neurovascular pedicle. Under direct vision, the pedicle can be readily isolated. If required, osteotomy of the foramen with a 2-mm osteotome is fast and straightforward. The cuts are U-shaped and directed medially, taking care that the osteotome does not slip downward into the canal.

Regarding secondary fistula closure, we shall proceed from posterior to anterior. A posterior fistula will require release of nasal mucoperiosteum as discussed above. Should it occur in the context of a failed Furlow repair, one may need import of posteriorly based buccinators flaps to fill the deficit. Fistulae in the midportion of the hard palate require isolation of nasal lining, turning it inward carefully. Advancement of mucoperiosteal for oral closure is assisted by an Alveolar Extension Palatoplasty (AEP) -type gingival release. The extra tissue harvested from the lingual margins makes AEP flaps wider that those produced by Von Langenbeck technique. ${ }^{[10]}$ When the fistula is anterior, AEP flaps need to be taken anteriorly, all the way to the cleft margin. Since the AEP flaps can be so readily mobilized, one should "cheat" when incising along the cleft margin. The incision can be designed to favour the oral side of the cleft by several millimetres, thus providing extra tissue to turn into the floor of the nose.

Finally, it is worth noting that the preponderance of patients in this paper presents as outside referrals (cleft camps, etc.). These patients are more likely to have recalcitrant fistulae. In many instances, their previous surgical management is unknown. In like manner, the fistula rate for this subset of patients is unknown. Those patients collected from within the SRMC system are a much smaller fraction of the total clefts performed. Their surgical treatment follows a known protocol. It would be interesting to separate out the distribution of clefts between the two groups to see if the fistulae seen in the SRMC versus those of the referral patients follow different patterns.

In summation, this reviewer would like to congratulate the
SRMC team for a careful analysis of palatal fistulae. The two groups represent very different surgical approaches (eclectic vs. standardized). Further study of this experience will undoubtedly prove rewarding. This commentary is designed to highlight common anatomic pitfalls in $\mathrm{CP}$ repair and how these can contribute to a fistula. Surgical techniques based upon an understanding of developmental anatomy are valuable to prevent fistula formation and to reconstruct fistulae when these are encountered.

\section{REFERENCES}

1. Landheer JA, Breugem CC, van der Molen M. Fistula incidence and predictors of fistula occurrence after cleft palate repair: Twostage closure versus one-stage closure. Cleft Palate Craniofac J 2010;47:623-30.

2. Lehner B, Wiltfang J, Strobel-Schwarthoff K, Benz M, Hirschfelder $\mathrm{U}$, Neukam FW. Influence of early hard palate closure in unilateral and bilateral cleft lip and palate on maxillary transverse growth during the first four years of life. Cleft Palate Craniofac J 2003;40:126-30.

3. Friede $\mathrm{H}$, Enemark $\mathrm{H}$. Long-term evidence for favorable midfacial growth after delated hard palate repair in UCLP patients. Cleft Palate Craniofac J 2001;38:323-9.

4. Nollett PJ, Katsaros C, van't Hof MA, Semb G, Shaw WC, Kuijper-Jagtman AM. Cephalometric evaluation of long-term craniofacial development in unilateral cleft lip and palate patients treated with delayed hard palate closure. Int J Oral Maxillofac Surg 2008;37:123-30.

5. Stein S, Dunsche A, Gellrich NC, Harle F, Jonas I. One- or twostage palate closure in patients with unilateral cleft lip and palate: Comparing cephalometric and occlusal outcomes. Cleft Palate Craniofac J 2007;44:13-22.

6. Carstens $\mathrm{MH}$. Neural tube programming and the pathogenesis of craniofacial clefts, part I: The neuromeric organization of the head and neck. Handb Clin Neurol 2007;87:247-76.

7. Carstens $\mathrm{MH}$. Neural tube programming and the pathogenesis of craniofacial clefts, part II: Mesenchyme, pharyngeal arches, developmental fields, and the assembly of the human face. Hanb Clin Neurol 2007;87:277-339.

8. Neuroembryology and functional anatomy of Cranio-facial Clefts 2009;42; 19-34.

9. Carstens $\mathrm{MH}$. Developmental field reassignment in unilateral cleft lip: Reconstruction of the premaxilla. In: Losee JE, Kirschner RE, editors. Comprehensive Cleft Care. New York: McGraw Hill; 2009. p. 241-66.

10. Carstens $\mathrm{MH}$. Sequential cleft management with the sliding sulcus technique and alveolar extension palatoplasty. J Craniofac Surg 1999;10:503-18. 